MKG-Chirurg 2012 · 5:5-5

DOI 10.1007/s12285-011-0275-6

Online publiziert: 12. Februar 2012

๑) Springer-Verlag 2012

\title{
Gemeinsame Stellungnahme der Herausgeberschaft und der DGMKG
}

In der grausamen Wirklichkeit bedeutete diese Einschätzung die Zwangssterilisation. Dass auch eine kontroverse bzw. empathische Haltung möglich war, beweisen Verhandlungen von Erbgesundheitsgerichten der Jahre 1934-1940, in denen bei einem Drittel der Fälle die Sterilisation des Betroffenen abgelehnt wurde. Bekannte Fachvertreter, wie Axhausen, Ernst und Rosenthal, haben sich eindeutig gegen Zwangsmaßnahmen bei Spaltpatienten positioniert, mussten allerdings für ihre Haltung persönliche Nachteile erleiden.

Die ideologische Verstrickung von Martin Waßmund müssen wir bedauernd zur Kenntnis nehmen und uns selbstverständlich die Frage stellen, warum wir erst mit einer derartig langen Verzögerung diesen Kenntnisstand erlangen konnten. Auch die Deutsche Gesellschaft für Chirurgie hat erst nach 65 Jahren die Kraft gewonnen, das Wirken ihrer Präsidenten in den Jahren 1933-1945 kritisch zu hinterfragen. Die Herausgeberschaft und die DGMKG danken Herrn PD Dr. Dr. Volker Thieme für die wissenschaftliche Aufarbeitung einer rassenhygienisch verblendeten wissenschaftlichen Debatte um die Entstehung von Spaltbildungen und der daraus resultierenden NS-Erbgesundheitspolitik. Wir dürfen Sie daher nachdrücklich bitten, sich sowohl mit der gedruckten „Kurzfassung“ als auch mit der „Online-Langfassung“ auseinanderzusetzen.
Herr Prof. Dr. Dr. Martin Waßmund kann trotz seiner unstrittigen Verdienste nicht mehr als untadeliges Vorbild und als Namensgeber des wissenschaftlichen Jahrespreises der DGMKG gelten. Konsequenterweise hat daher der Vorstand der DGMKG am 23.11.2011 einstimmig die Umbenennung in „Wissenschaftspreis der DGMKG“ beschlossen. Diese Entscheidung resultiert aus dem uneingeschränkten Bekenntnis für ein hohes ethisches Anforderungsprofil zum Schutz des menschlichen Lebens in all seinen Erscheinungsformen.

Prof. Dr. Dr. Elmar Esser,

Schriftleitung

Dr. Erich-Theo Merholz, Präsident DGMKG

Prof. Dr. Dr. Alexander Hemprich, Vizepräsident DGMKG 